KEK-TH-736

Dec. 2000

\title{
High Energy Behavior of Wilson Lines
}

\author{
Avinash Dhar 周, Yoshihisa Kitazawaf \\ Laboratory for Particle and Nuclear Physics, \\ High Energy Accelerator Research Organization (KEK), \\ Tsukuba, Ibaraki 305-0801, Japan
}

\begin{abstract}
We investigate the high energy behavior of the correlation functions of the open Wilson lines in noncommutative gauge theory. We obtain a very simple physical picture that they are bound to form a group of closed Wilson loops. We prove our claim in the weak coupling region by perturbative analysis. We emphasize the importance of respecting the cyclic symmetry of the straight Wilson lines to compute the correlation functions. The implications for stringy calculation of the correlators are also discussed.
\end{abstract}

\footnotetext{
* On leave from Dept of Theoretical Phys, Tata Institute, Mumbai 400005, INDIA.

$\dagger$ e-mail address : adhar@post.kek.jp

$\ddagger$ e-mail address : kitazawa@post.kek.jp
} 


\section{Introduction}

In matrix model formulation of superstring theory, not only matter but also space [1] or even space-time 2] may emerge out of matrices. Noncommutative gauge theory may be regarded as a concrete realization of such a possibility [3]. We can obtain fully noncommutative gauge theory by expanding IIB matrix model around noncommutative backgrounds [4]. In fact such a matrix model construction has been very useful to elucidate physical properties of noncommutative gauge theory [10]. It has further been argued that four dimensional noncommutative gauge theory with maximal SUSY $\left(N C Y M_{4}\right)$ may be interpreted as superstring theory with noncommutativity scale as its effective string scale[6]. It may serve as a testing ground for the proposal that matrix models may serve as nonperturbative formulation of superstring theory.

The gauge invariant observables in noncommutative gauge theory involve not only closed Wilson loops but also open Wilson loops (Wilson lines) [5] [1] [12] [19]. It has also been pointed out that the operators which couple to graviton multiplets may be constructed through them [5]. The validity of such an argument is confirmed by the recent investigations [14] [15]. These authors have also shown that the Wilson lines are closely related to the Seiberg-Witten map[9].

The correlators of the Wilson lines are found to exhibit stringy exponential suppression behavior at high energy limit [11]. In our recent work, we have investigated the strong coupling behavior of the expectation value of the Wilson loops 13. Our proposal is to consider Nambu-Goto action in the metric of the corresponding supergravity solution. The four dimensional part of the metric is conformally flat while the conformal factor possesses the unique maximum in the radial coordinate. We postulate that the Wilson loops in $N C Y M_{4}$ may be represented by the closed contours at the the maximum of the space-time metric. In our proposal, the expectation value of the Wilson loops are given by the extremum of the Nambu-Goto action, namely the minimum area. We have further mentioned that the high energy limit of the Wilson line correlators may be identical to the expectation value of the large Wilson loops.

In this paper we construct a proof of the above stated equivalence. We first give an heuristic argument based on the loop equations in section 2. In section 3, we prove the equivalence by the perturbative analysis of the correlators in the weak coupling region.

\footnotetext{
${ }^{1}$ NCYM is equivalent to large $N$ twisted reduced model [20] 22] 22]. See also [23].
} 
Since our argument based on the loop equation holds in both the weak and strong coupling regions, we expect that the equivalence does not break down even in the strong coupling regime. If so, our recent work in 113 becomes relevant to this problem. In the concluding section, we discuss possible strong coupling behavior of the Wilson line correlators in the high energy limit based on such a line of argument.

\section{Loop equations in NCYM}

In this section, we briefly review matrix model constructions of NCYM first [4. We recall IIB matrix model action:

$$
S=-\frac{1}{g^{2}} \operatorname{Tr}\left(\frac{1}{4}\left[A_{\mu}, A_{\nu}\right]\left[A^{\mu}, A^{\nu}\right]+\frac{1}{2} \bar{\psi} \Gamma^{\mu}\left[A_{\mu}, \psi\right]\right) .
$$

Here $\psi$ is a ten dimensional Majorana-Weyl spinor field, and $A_{\mu}$ and $\psi$ are $N \times N$ Hermitian matrices. The noncommutative Yang-Mills theory is obtained by expanding the theory around the following background:

$$
\left[\hat{p}_{\mu}, \hat{p}_{\nu}\right]=i B_{\mu \nu}
$$

where $B_{\mu \nu}$ are $c$-numbers. We assume the rank of $B_{\mu \nu}$ to be $\tilde{d}$ and define its inverse $C^{\mu \nu}$ in $\tilde{d}$ dimensional subspace. We expand $A_{\mu}=\hat{p}_{\mu}+\hat{a}_{\mu}$. Noncommutative Yang-Mills can be realized through matrix models by the the following map from matrices onto functions

$$
\begin{aligned}
\hat{a} & \rightarrow a(x), \\
\hat{a} \hat{b} & \rightarrow a(x) \star b(x), \\
T r & \rightarrow \sqrt{\operatorname{det} B}\left(\frac{1}{2 \pi}\right)^{\frac{\tilde{d}}{2}} \int d^{\tilde{d}} x, \\
g^{2} & \rightarrow \sqrt{\operatorname{det} B}\left(\frac{1}{2 \pi}\right)^{\frac{\tilde{d}}{2}} g_{N C}^{2} .
\end{aligned}
$$

The gauge invariant observables in NCYM are the Wilson loops:

$$
W(C)=\operatorname{Tr}\left[\operatorname{Pexp}\left(i \int_{C} d x^{\mu}(\sigma) A_{\mu}\right)\right]
$$

where $C$ denotes a contour parametrized by $x^{\mu}(\sigma)$. Let us consider the following correlation functions:

$$
\int d A d \psi \operatorname{Tr}\left[t^{a} P \exp \left(i \int_{C_{1}} d x^{\mu}(\sigma) A_{\mu}\right)\right] W\left(C_{2}\right) e^{-S} .
$$

We consider the following infinitesimal change of the variables as $A_{\mu} \rightarrow A_{\mu}+\epsilon t^{a}$. By using the completeness condition of the generators of $U(N)$ :

$$
\sum_{a} t_{i j}^{a} t_{k l}^{a}=\delta_{i l} \delta_{j k}
$$


we obtain,

$$
\begin{aligned}
& <\frac{1}{g^{2}} \operatorname{Tr}\left[\left\{\left[A_{\mu},\left[A_{\mu}, A_{\nu}\right]\right]+\frac{1}{2} \Gamma_{\nu}[\psi, \bar{\psi}]_{+}\right\} \operatorname{Pexp}\left(i \int_{C_{1}} d x^{\mu}\left(\sigma_{1}\right) A_{\mu}\right)\right] W\left(C_{2}\right)> \\
= & i \int_{0}^{1} d t \dot{x}^{\nu}<\operatorname{Tr}\left[\operatorname{Pexp}\left(i \int_{0}^{t} d \sigma_{1} \dot{x}^{\mu} A_{\mu}\right)\right] \operatorname{Tr}\left[\operatorname{Pexp}\left(i \int_{t}^{1} d \sigma_{1} \dot{x}^{\mu} A_{\mu}\right)\right] W\left(C_{2}\right)> \\
+ & i \int_{0}^{1} d s \dot{x}^{\nu}<\operatorname{Tr}\left[\operatorname{Pexp}\left(i \int_{0}^{s} d \sigma_{2} \dot{x}^{\mu} A_{\mu}\right) \exp \left(i \int_{C_{1}} d x^{\mu}\left(\sigma_{1}\right) A_{\mu}\right) \exp \left(i \int_{s}^{1} d \sigma_{2} \dot{x}^{\mu} A_{\mu}\right)\right]>.
\end{aligned}
$$

The first and second term on the right-hand side of the above equation represents the splitting and joining of the loops respectively 8$]$.

The Wilson loops in the matrix model are mapped to those in NCYM by eq.(2.3):

$$
\begin{aligned}
& \operatorname{Tr}\left[\operatorname{Pexp}\left(i \int_{C} d x^{\mu}(\sigma) A_{\mu}\right)\right] \\
= & \left.\sqrt{\operatorname{det} B}\left(\frac{1}{2 \pi}\right)^{\frac{\tilde{d}}{2}} \int d^{\tilde{d}} x \operatorname{Pexp}\left(i \int_{C} d y^{\mu}(\sigma) a_{\mu}(x+y(\sigma))\right) \exp \left(i k^{\mu} x_{\mu}\right)\right)_{\star}
\end{aligned}
$$

where the total momentum $k_{\mu}$ is related to the vector $d^{\mu}$ which connects the two ends of the contour as $k_{\mu}=B_{\mu \nu} d^{\nu}$. The symbol $\star$ in the above expression reminds us that all products of fields must be understood as $\star$ products. In order to prove the above identity we consider the following discretization of a Wilson loop.

$$
P \exp \left(i \int_{C} d x^{\mu}(\sigma) A_{\mu}\right)=\lim _{n \rightarrow \infty} \prod_{j=1}^{n} \exp \left(i \Delta x_{j} \cdot(\hat{p}+\hat{a})\right) .
$$

The above quantity can be rewritten as

$$
\begin{aligned}
& \lim _{n \rightarrow \infty} \prod_{j=1}^{n} \exp \left(i \Delta x_{j} \cdot \hat{p}\right) \exp \left(i \Delta x_{j} \hat{a}\right) \\
= & \lim _{n \rightarrow \infty}\left(\prod_{j=1}^{n} V_{j} \exp \left(i \Delta x_{j} \hat{a}\right) V_{j}^{\dagger}\right) V_{n} \\
= & \lim _{n \rightarrow \infty}\left(\prod_{j=1}^{n} \exp \left(i \Delta x_{j} V_{j} \hat{a} V_{j}^{\dagger}\right)\right) V_{n}
\end{aligned}
$$

where $V_{j}=\left(\prod_{k=1}^{j} \exp \left(i \Delta x_{k} \hat{p}\right)\right.$ is the translation operator along the contour $C$ from the first to the $j$-th segment. After applying eq.(2.3) to the above and taking $n \rightarrow \infty$ limit of it, we obtain the right-hand side of eq.(2.8). We then obtain the Schwinger-Dyson equations in NCYM [16]

$$
<\int d^{\tilde{d}} x\left[\frac{1}{i}\left\{\left[D_{\mu},\left[D_{\mu}, D_{\nu}\right]\right]+\frac{1}{2} \Gamma_{\nu}[\psi, \bar{\psi}]_{+}\right\}\right.
$$




$$
\begin{aligned}
& \left.\times \operatorname{Pexp}\left(i \int_{C_{1}} d y^{\mu}\left(\sigma_{1}\right) a_{\mu}(x+y(\sigma)) \exp \left(i k_{1} \cdot x\right)\right)\right]_{\star} W\left(C_{2}\right)> \\
= & g_{N C}^{2} \operatorname{det} B\left(\frac{1}{2 \pi}\right)^{\tilde{d}} \int_{0}^{1} d t \dot{x}^{\nu}<\int d^{\tilde{d}} x_{1}\left[\operatorname{Pexp}\left(i \int_{C} d y^{\mu}\left(\sigma_{1}\right) a_{\mu}\left(x_{1}+y\left(\sigma_{1}\right)\right) \exp \left(i k \cdot x_{1}\right)\right)\right]_{\star} \\
& \times \int d^{\tilde{d}} x_{2}\left[P \exp \left(i \int_{C_{1}-C} d y^{\mu}\left(\sigma_{2}\right) a_{\mu}\left(x_{2}+y\left(\sigma_{2}\right)\right) \exp \left(i\left(k_{1}-k\right) \cdot x_{2}\right)\right)\right]_{\star} W\left(C_{2}\right)> \\
+ & g_{N C}^{2} \int_{0}^{1} d s \dot{x}^{\nu}<\int d^{\tilde{d}} x\left[P \exp \left(i \int_{\tilde{C}} d y^{\mu}\left(\sigma_{1}\right) a_{\mu}\left(x+y\left(\sigma_{1}\right)\right) \exp (i \tilde{k} \cdot x)\right)\right. \\
& \left.\times \operatorname{Pexp}\left(i \int_{C_{1}} d y^{\mu}\left(\sigma_{1}\right) a_{\mu}(x+y(\sigma))\right) \exp \left(i k_{1}^{\mu} x_{\mu}\right)\right) \\
& \left.\times \operatorname{Pexp}\left(i \int_{C_{2}-\tilde{C}} d y^{\mu}\left(\sigma_{2}\right) a_{\mu}\left(x+y\left(\sigma_{2}\right)\right) \exp \left(i\left(k_{2}-\tilde{k}\right) \cdot x\right)\right)\right]_{\star}>
\end{aligned}
$$

We may generalize the above equation in $U(1)$ gauge group to $U(m)$ by considering the direct product of noncommutative space-time and gauge group which can be realized in $U(N m)$. We consider the generators of $U(N m)$ which satisfy

$$
\sum_{a} \sum_{b} t_{i j}^{a} \lambda_{\alpha \beta}^{b} t_{k l}^{a} \lambda_{\gamma \delta}^{b}=\delta_{i l} \delta_{j k} \delta_{\alpha \delta} \delta_{\beta \gamma}
$$

where $\lambda^{b}$ denotes the generators of $U(m)$. The Wilson loop becomes

$$
\begin{aligned}
& \frac{1}{m} \operatorname{Tr}\left[\operatorname{Pexp}\left(i \int_{C} d x^{\mu}(\sigma) A_{\mu}\right)\right] \\
= & \frac{1}{m} \sqrt{\operatorname{det} B}\left(\frac{1}{2 \pi}\right)^{\frac{\tilde{d}}{2}} \int d^{\tilde{d}} x \operatorname{tr}\left[\operatorname{Pexp}\left(i \int_{C} d y^{\mu}(\sigma) a_{\mu}(x+y(\sigma))\right) \exp \left(i k^{\mu} x_{\mu}\right)\right]_{\star} .
\end{aligned}
$$

Following the analogous steps as in $U(1)$ case, we obtain

$$
\begin{aligned}
& <\frac{1}{m} \int d^{\tilde{d}} x \operatorname{tr}\left[\frac{1}{i}\left\{\left[D_{\mu},\left[D_{\mu}, D_{\nu}\right]\right]+\frac{1}{2} \Gamma_{\nu}[\psi, \bar{\psi}]_{+}\right\}\right. \\
& \left.\times \operatorname{Pexp}\left(i \int_{C_{1}} d y^{\mu}\left(\sigma_{1}\right) a_{\mu}(x+y(\sigma)) \exp \left(i k_{1} \cdot x\right)\right)\right]_{\star} W\left(C_{2}\right)> \\
= & \lambda \operatorname{det} B\left(\frac{1}{2 \pi}\right)^{\tilde{d}} \frac{1}{m^{2}} \int_{0}^{1} d t \dot{x}^{\nu}<\int d^{\tilde{d}} x_{1} \operatorname{tr}\left[\operatorname{Pexp}\left(i \int_{C} d y^{\mu}\left(\sigma_{1}\right) a_{\mu}\left(x_{1}+y\left(\sigma_{1}\right)\right) \exp \left(i k \cdot x_{1}\right)\right)\right]_{\star} \\
& \times \int d^{\tilde{d}} x_{2} \operatorname{tr}\left[\operatorname{Pexp}\left(i \int_{C_{1}-C} d y^{\mu}\left(\sigma_{2}\right) a_{\mu}\left(x_{2}+y\left(\sigma_{2}\right)\right) \exp \left(i\left(k_{1}-k\right) \cdot x_{2}\right)\right)\right]_{\star} W\left(C_{2}\right)> \\
+ & \frac{\lambda}{m^{2}} \int_{0}^{1} d s \dot{x}^{\nu} \frac{1}{m}<\int d^{\tilde{d}} x \operatorname{tr}\left[\operatorname{Pexp}\left(i \int_{\tilde{C}} d y^{\mu}\left(\sigma_{1}\right) a_{\mu}\left(x+y\left(\sigma_{1}\right)\right) \exp (i \tilde{k} \cdot x)\right)\right. \\
& \left.\times \operatorname{Pexp}\left(i \int_{C_{1}} d y^{\mu}\left(\sigma_{1}\right) a_{\mu}(x+y(\sigma))\right) \exp \left(i k_{1}^{\mu} x_{\mu}\right)\right) \\
& \left.\times \operatorname{Pexp}\left(i \int_{C_{2}-\tilde{C}} d y^{\mu}\left(\sigma_{2}\right) a_{\mu}\left(x+y\left(\sigma_{2}\right)\right) \exp \left(i\left(k_{2}-\tilde{k}\right) \cdot x\right)\right)\right]_{\star}>
\end{aligned}
$$

where $t r$ denotes the trace operation over $U(m)$ and $\lambda=g_{N C}^{2} m$ is the 't Hooft coupling.

The correlation functions of the Wilson lines can be investigated in principle by solving these loop equations. In this paper we show that the correlators of the very long Wilson 
lines are identical to the expectation value of the Wilson loop which can be formed from them. Since very long Wilson lines carry large momenta, it is the equivalence which holds in the high energy limit. In fact such an equivalence can be expected from the loop equations.

Let us consider the simplest looking loop equation eq.(2.7). The first term and the second term on the right hand side represent splitting and joining terms respectively. For long straight Wilson lines, we can argue that the joining term is dominant since it is proportional to the length of $C_{2}$. Since the left-hand side of the equation is essentially the deformation of the first Wilson line $C_{1}$, we can argue that the expectation value of Wilson lines is equivalent to that of completely joined Wilson lines namely closed Wilson loop. In the next section, we construct a perturbative proof of the equivalence which is in accord with the loop equation argument.

\section{Wilson lines as Wilson loops}

In NCYM, we also have the gauge invariant operators specified by the open contours as is defined in eq.(2.8). Let us consider the two point function of $W\left(C_{1}\right)$ and $W\left(C_{2}\right)$ for example. Two contours $C_{1}$ and $C_{2}$ specify the shapes of two open segments. Let us denote the vector which connects the two ends of a contour by $\vec{d}$. In noncommutative Yang-Mills, such an open segment carries the momentum $\vec{k}_{\mu}=B_{\mu \nu} \vec{d}^{\nu}$. In order for two point functions to be nonvanishing, $W\left(C_{1}\right)$ and $W\left(C_{2}\right)$ must possess vanishing total momentum. In other words, $C_{1}$ and $C_{2}$ can be put together to form a closed loop. Let us denote the vector which connects the tail of $C_{1}$ and head of $C_{2}$ as $\vec{x}$. What is calculated in noncommutative Yang-Mills theory can be expressed as

$$
<W\left(C_{1}\right) W\left(C_{2}\right)>=V \int d^{4} \operatorname{xexp}(i \vec{k} \cdot \vec{x})<W\left(C_{1}, C_{2}, \vec{x}\right)>
$$

Namely it can be interpreted in terms of the expectation value of the operator specified by a closed loop which contains two segments $C_{1}$ and $C_{2}$. However $<W\left(C_{1}, C_{2}, \vec{x}\right)>$ cannot be obtained by a simple Fourier transformation of $\left\langle W\left(C_{1}\right) W\left(C_{2}\right)>\right.$, since the contours $C_{1}$ and $C_{2}$ depend on $\vec{k}$.

In fact we can express the two point function of the Wilson loops in terms of the one point function through matrix model constructions. If the gauge group is $U(1)$, we obtain

$$
\operatorname{Tr}\left[\operatorname{Pexp}\left(i \int_{C_{1}} d x^{\mu}(\sigma) A_{\mu}\right)\right] \operatorname{Tr}\left[\operatorname{Pexp}\left(i \int_{C_{2}} d x^{\mu}(\sigma) A_{\mu}\right)\right]
$$




$$
=\frac{1}{N} \sum_{\vec{l}} \operatorname{Tr}\left[\operatorname{Pexp}\left(i \int_{C_{1}} d x^{\mu}(\sigma) A_{\mu}\right) \exp (i \vec{l} \cdot \hat{p}) \operatorname{Pexp}\left(i \int_{C_{2}} d x^{\mu}(\sigma) A_{\mu}\right) \exp (-i \vec{l} \cdot \hat{p})\right]
$$

where we have used the completeness condition of the generators of $U(N)$.

$$
\frac{1}{N} \sum_{\vec{l}} \exp (i \vec{l} \cdot \hat{p})_{a, b} \exp (-i \vec{l} \cdot \hat{p})_{c, d}=\delta_{a, d} \delta_{b, c}
$$

If the gauge group is $U(m)$, we either keep the trace operation over $U(m)$ in each Wilson line or insert the generators of $U(m)\left(\lambda^{a}\right)$ such as

$$
\begin{aligned}
& \operatorname{Tr}\left[\operatorname{Pexp}\left(i \int_{C_{1}} d x^{\mu}(\sigma) A_{\mu}\right)\right] \operatorname{Tr}\left[\operatorname{Pexp}\left(i \int_{C_{2}} d x^{\mu}(\sigma) A_{\mu}\right)\right] \\
= & \sum_{a} \frac{1}{N} \sum_{\vec{l}} \operatorname{Tr}\left[\operatorname{Pexp}\left(i \int_{C_{1}} d x^{\mu}(\sigma) A_{\mu}\right) \lambda^{a} \exp (i \vec{l} \cdot \hat{p})\right. \\
& \left.\times \operatorname{Pexp}\left(i \int_{C_{2}} d x^{\mu}(\sigma) A_{\mu}\right) \lambda^{a} \exp (-i \vec{l} \cdot \hat{p})\right] .
\end{aligned}
$$

The matrix model constructions such as eq.(3.2) can be translated into noncommutative gauge theory expressions as follows:

$$
\begin{aligned}
& \exp (i \Phi) \int d^{4} x \int d^{4} \tilde{x} \exp \left(i k^{\mu} \tilde{x}_{\mu}\right) \\
& {\left[P \exp \left(i \int_{C_{1}} d y_{1}^{\mu}(\sigma) a_{\mu}\left(x+y_{1}(\sigma)\right)\right) P \exp \left(i \int_{C_{2}} d y_{2}^{\mu}(\sigma) a_{\mu}\left(x+\tilde{x}+y_{2}(\sigma)\right)\right)\right]_{\star}}
\end{aligned}
$$

where $\Phi$ is the magnetic flux enclosed in the closed loop $C_{1}+C_{2}$. It is given by the area of the loop divided by $C$.

We next consider the three point function.

$$
\begin{aligned}
& \operatorname{Tr}\left[\operatorname{Pexp}\left(i \int_{C_{1}} d x^{\mu}(\sigma) A_{\mu}\right)\right] \operatorname{Tr}\left[\operatorname{Pexp}\left(i \int_{C_{2}} d x^{\mu}(\sigma) A_{\mu}\right)\right] \operatorname{Tr}\left[\operatorname{Pexp}\left(i \int_{C_{3}} d x^{\mu}(\sigma) A_{\mu}\right)\right] \\
= & \frac{1}{N} \sum_{\vec{l}} \frac{1}{N} \sum_{\vec{m}} \operatorname{Tr}\left[\operatorname{Pexp}\left(i \int_{C_{1}} d x^{\mu}(\sigma) A_{\mu}\right) \exp (i \vec{l} \cdot \hat{p}) \operatorname{Pexp}\left(i \int_{C_{2}} d x^{\mu}(\sigma) A_{\mu}\right)\right. \\
& \left.\times \exp (i \vec{m} \cdot \hat{p}) \operatorname{Pexp}\left(i \int_{C_{2}} d x^{\mu}(\sigma) A_{\mu}\right) \exp (-i \vec{m} \cdot \hat{p}) \exp (-i \vec{l} \cdot \hat{p})\right]
\end{aligned}
$$

It can be translated into noncommutative gauge theory as

$$
\begin{aligned}
& \exp (i \Phi) \int d^{4} x \int d^{4} x_{2} \int d^{4} x_{3} \exp \left(i\left(k_{2}+k_{3}\right) \cdot x_{2}+i k_{3} \cdot x_{3}\right) \\
& {\left[P \exp \left(i \int_{C_{1}} d y^{\mu}(\sigma) a_{\mu}(x+y(\sigma))\right) \exp \left(i \int_{C_{2}} d y^{\mu}(\sigma) a_{\mu}\left(x+x_{2}+y(\sigma)\right)\right)\right.} \\
& \left.\times \exp \left(i \int_{C_{3}} d y^{\mu}(\sigma) a_{\mu}\left(x+x_{2}+x_{3}+y(\sigma)\right)\right)\right]_{\star}
\end{aligned}
$$


where $x_{2}$ connects the tail of $C_{1}$ and head of $C_{2}$ and so does $x_{3}$ between $C_{2}$ and $C_{3}$. In the case of $n$ point functions of the Wilson lines, the prescription is just analogous. What is calculated in noncommutative gauge theory is

$$
\begin{aligned}
& <W\left(C_{1}\right) W\left(C_{2}\right) \cdots W\left(C_{n}\right)> \\
= & V \prod_{i=1}^{n} \int d^{4} x_{i} \exp \left(i \sum_{i=2}^{i=n} \vec{l}_{i} \cdot \vec{x}_{i}\right)<W\left(C_{1}, C_{2}, \cdots, C_{n} ; \vec{x}_{1}, \vec{x}_{2}, \cdots, \vec{x}_{n-1}\right)>.
\end{aligned}
$$

In this expression $x_{1}$ denotes the center of mass coordinate of the system. The vector $\vec{x}_{i}(i \geq 2)$ connects the tail of $C_{i-1}$ and the head of $C_{i}$ and $l_{i}=\sum_{j=i}^{j=n} k_{j}$.

The real space correlator can be regarded as a one point function of the operator of a single closed loop. The loop consists of the segments $C_{i}$ which are accompanied with the gauge fields. These segments are connected by straight segments of length $\left|\vec{x}_{i}\right|$ which do not involve gauge fields. Although these are new types of the operators which are distinct from closed Wilson loops, they are not gauge invariant observables by themselves. Here we would like to point out that such an object is useful to discuss the correlation functions of long Wilson lines which carry large momenta. In such a region, we can show that the correlators of the Wilson lines are indistinguishable from those of large closed Wilson loops. In what follows we prove our contention in the weak coupling region by using the perturbation theory.

Let us consider the leading contribution to the two point function of straight Wilson lines.

$$
\begin{aligned}
& \int d^{4} x \int d^{4} x_{1} \exp \left(i k \cdot x_{1}\right) \\
& <P \int_{C_{1}} d a \cdot A\left(x+x_{1}+a\right) \star \int_{C_{2}} d b_{1} \cdot A(x+b)> \\
= & V \int d a d b \exp (-i k \cdot(a-b)) \frac{g_{N C}^{2}}{k^{2}}
\end{aligned}
$$

where $V$ denotes the space-time volume. $a$ and $b$ denote the locations of the two ends of a propagator on the Wilson lines $C_{1}$ and $C_{2}$ respectively. We also use $A(x)$ to denote gauge fields in the remaining of this section. This expression is invariant under independent translations of $a$ and $b$ along the contours of the Wilson lines. It is the manifestation of the cyclic symmetry of the straight Wilson lines. Due to this symmetry we can fix $a=b=0$ after factoring out the volume factor $C k_{1} C k_{2}$. Since $x_{1} \sim 1 / k$, we find that the lowest order contribution represents a long rectangle of the horizontal length $C k$ and the vertical length of $1 / k$. 
We move on to the three point functions in eq.(3.7). In the weak coupling region, we may retain

$$
\begin{aligned}
& \exp (i \Phi) \int d^{4} x \int d^{4} x_{1} \int d^{4} x_{3} \exp \left(i k_{1} \cdot x_{1}+i k_{3} \cdot x_{3}\right) \\
& <P \int_{C_{1}} d a \cdot A\left(x+x_{1}+a\right) \star \int_{C_{2}} d b_{1} \cdot A\left(x+b_{1}\right) \\
& \star \int_{C_{2}} d b_{2} \cdot A\left(x+b_{2}\right) \star \int_{C_{3}} d c \cdot A\left(x+x_{3}+c\right)>
\end{aligned}
$$

where we have only considered the amplitude where gluons are exchanged between $\left(C_{1}, C_{2}\right)$ and $\left(C_{2}, C_{3}\right)$. We also need to add three other types of contributions to obtain the full amplitude at the tree level. In this particular example, we have not only the ordered contributions with $b_{1}<b_{2}$ but also the other ordering $b_{2}<b_{1}$ where by $b_{1}$ we imply the location of the propagator with momentum $k_{1}$. The latter contribution can be reexpressed as

$$
\begin{aligned}
& \exp \left(i k_{3} \cdot\left(x+b_{2}\right)\right) \star \exp \left(i k_{1} \cdot\left(x+b_{1}\right)\right) \\
= & \exp \left(i k_{1} \cdot\left(x+b_{1}\right)\right) \star \exp \left(i k_{3} \cdot\left(x+b_{2}+C k_{2}\right)\right) .
\end{aligned}
$$

In this expression $\tilde{b}_{2}=b_{2}+C k_{2}$ is such that $b_{1}<\tilde{b}_{2}<b_{1}+C k_{2}$. It can be shown to be independent of $b_{1}$ after changing the variable from $b_{2}$ to $\tilde{b}_{2}$.

We note here that eq.(3.10) is invariant under independent translations of $a$ along $C_{1}$ and $c$ along $C_{3}$. It is also invariant under the simultaneous translations of $b_{1}, b_{2}$ along $C_{2}$. Due to the symmetry, we can fix the location of $a, b_{1}, c$ at the ends of the Wilson line after factoring out the volume factor $C k_{1} C k_{2} C k_{3}$. In this way, we obtain

$$
\begin{aligned}
& V g_{N C}^{4} \exp \left(\frac{i}{2} k_{1} C k_{2} \sin \left(\theta_{1}\right)\right) \cos \left(\theta_{1}\right) \cos \left(\theta_{3}\right) \frac{1}{k_{1}{ }^{2}} \frac{1}{k_{3}{ }^{2}} C k_{1} C k_{2} C k_{3} \\
& \int_{0}^{C k_{2}} d\left|b_{2}\right| \exp \left(-i k_{1} \sin \left(\theta_{1}\right)\left|b_{2}\right|\right) \\
= & V g_{N C}^{4} \cot \left(\theta_{1}\right) \cos \left(\theta_{3}\right) \frac{1}{k_{1}{ }^{2}} \frac{1}{k_{3}{ }^{2}} \frac{1}{i} C C k_{2} C k_{3} \\
& \left\{\exp \left(\frac{i}{2} k_{1} C k_{2} \sin \left(\theta_{1}\right)\right)-\exp \left(-\frac{i}{2} k_{1} C k_{2} \sin \left(\theta_{1}\right)\right)\right\}
\end{aligned}
$$

where $\theta_{1}$ and $\theta_{3}$ are the angles of the two corners of the triangle formed by $\left(C_{1}, C_{2}\right)$ and $\left(C_{2}, C_{3}\right)$ respectively.

We emphasize here that straight Wilson lines possess the cyclic symmetry. In fact the Wilson lines can be written as $\operatorname{Tr}\left(U^{n}\right)$ in the matrix model construction where $U=\exp (i \Delta x$. $(\hat{p}+\hat{a}))$ and $\Delta x=C k / n$. In order to evaluate the correlation functions which involve a 
particular Wilson line, we need to attach propagators with momenta $\left\{p_{i}\right\}$ to it. It is clear that there are $n$ ways to pick the first gauge field with momentum $p_{1}$ from $\operatorname{Tr}\left(U^{n}\right)$. They are all equivalent due to the cyclic symmetry of the trace. If we connect two propagators, only the relative position of the second one to the first matters due to the cyclic symmetry. So in general the correlator is proportional to the length of the Wilson line and we can fix the location of one of the propagators.

The propagators are very short in the large $k$ limit since their lengths are of $O(1 / k)$. Furthermore the $\left|b_{2}\right|$ integration is only supported by the two infinitesimal segments of the width $O(1 / k)$ at the boundaries of the integration region. Therefore in the large $k$ limit, the first term on the right-hand side of eq.(3.12) corresponds to a closed triangle configuration made of $\left(C_{1}, C_{2}, C_{3}\right)$ in that order. The second term appears to represent the configuration where three Wilson lines share the same end point. However we need to recall the cyclic symmetry of the Wilson lines. The points on the same Wilson line can be freely moved simultaneously. In the case of two points on the same Wilson line, a pair of vanishingly close points $\left(b_{1}, b_{2}\right)$ is equivalent to the pair of the end points $\left(b_{2}, b_{1}\right)$ due to the cyclic symmetry. In this way we can interpret it as the closed triangle made of $\left(C_{2}, C_{1}, C_{3}\right)$ in that order. Therefore we can indeed see that the Wilson lines are bound to form closed Wilson loops in the large $k$ limit in the lowest order of perturbation theory. In the three point function case, we find two different triangles which can be formed by the Wilson lines. We can also understand the phase in each term $\pm k_{1} C k_{2} \sin \left(\theta_{1}\right) / 2$ as the magnetic flux passing through the respective triangle. Although there is a topologically distinct diagram which involves the three point vertices at the tree level, the structure of such a contribution can be shown to be just analogous.

We can extend similar analysis to $n$ point functions of the Wilson lines. By such an analysis we can easily convince ourselves that the $n$ point functions of Wilson lines are effectively described by a group of Wilson loops which can be formed from the Wilson lines. It is certainly clear that the correlation function is saturated by a finite numbers of configurations for $n$ point functions just like the three point function case. We argue that they could only be Wilson loops due to the gauge invariance.

In the weak coupling region, we might argue that the investigation of the tree diagrams suffices. However it is certainly not so for two point functions[11]. It is found that the leading contribution at the $n$-th order is $(\lambda|k||C k| / 4 \pi)^{n} /(n !)^{2}$. The summation over $n$ can be estimated by the saddle point method as $\exp (\sqrt{\lambda|k||C k| / \pi})$. The average separation of 
the two Wilson lines is found to be $\langle n>/ k \sim \sqrt{\lambda|C k| /|k|}$. Although it is much larger than the tree level estimate $1 / k$, it is still much smaller than the noncommutativity scale in the weak coupling regime.

In the case of three point functions, we also find logarithmic divergences in association of the corners. The first example occurs at the next order.

$$
\begin{aligned}
& \exp (i \Phi) \int d^{4} x \int d^{4} x_{1} \int d^{4} x_{3} \exp \left(i k_{1} \cdot x_{1}+i k_{3} \cdot x_{3}\right) \\
& P \int_{C_{1}} d a \cdot A\left(x+x_{1}+a_{2}\right) \star \int_{C_{1}} d a \cdot A\left(x+x_{1}+a_{1}\right) \\
& \star \int_{C_{2}} d b_{1} \cdot A\left(x+b_{1}\right) \star \int_{C_{2}} d b_{2} \cdot A\left(x+b_{2}\right) \star \int_{C_{2}} d b_{3} \cdot A\left(x+b_{3}\right) \star \int_{C_{3}} d c \cdot A\left(x+x_{3}+c\right) \\
= & \exp (i \Phi) \int d^{4} x \int d^{4} x_{1} \int d^{4} x_{3} \exp \left(i k_{1} \cdot x_{1}+i k_{3} \cdot x_{3}\right) \\
& \times \int_{C_{1}} d a_{1} \cdot \int_{C_{2}} d b_{1} \int_{C_{1}} d a_{2} \cdot \int_{C_{2}} d b_{2} \int_{C_{2}} d b_{3} \cdot \int_{C_{3}} d c \\
& \frac{m g_{N C}^{2}}{4 \pi^{2}\left(x_{1}+a_{1}-b_{1}\right)^{2}} \frac{g_{N C}^{2}}{4 \pi^{2}\left(x_{1}+a_{2}-b_{2}\right)^{2}} \frac{m \pi^{2}\left(x_{3}+c-b_{3}\right)^{2}}{=} \\
& V \frac{\lambda^{3}}{m^{2}} \exp (i \Phi) \int_{C_{1}} d a_{1} \cdot \int_{C_{2}} d b_{1} \int_{C_{1}} d a_{2} \cdot \int_{C_{2}} d b_{2} \int_{C_{2}} d b_{3} \cdot \int_{C_{3}} d c \\
& \int \frac{d^{4} p}{(2 \pi)^{4}} \frac{1}{\left(k_{1}-p\right)^{2}} \frac{1}{p^{2}} \frac{1}{k_{3}{ }^{2}} \exp \left(-i\left(k_{1}-p\right) \cdot\left(a_{1}-b_{1}\right)-i p \cdot\left(a_{2}-b_{2}\right)-i k_{3} \cdot\left(c-b_{3}\right)\right) .
\end{aligned}
$$

We can now fix $a_{1}=b_{1}=c=0$ due to the cyclic symmetry of the Wilson lines.

$$
\begin{aligned}
& V \frac{\lambda^{3}}{m^{2}} \exp (i \Phi) \cos \left(\theta_{1}\right) \cos \left(\theta_{3}\right) C k_{1} C k_{2} C k_{3} \int_{C_{1}} d a_{2} \cdot \int_{C_{2}} d b_{2} \int_{C_{2}} d\left|b_{3}\right| \\
& \int \frac{d^{4} p}{(2 \pi)^{4}} \frac{1}{\left(k_{1}-p\right)^{2}} \frac{1}{p^{2}} \frac{1}{k_{3}^{2}} \exp \left(-i p \cdot\left(a_{2}-b_{2}\right)+i k_{3} \cdot b_{3}\right) \\
= & V i \frac{\lambda^{3}}{m^{2}} \exp (i \Phi) \cos ^{2}\left(\theta_{1}\right) \cot \left(\theta_{3}\right) C k_{1} C k_{2} C \int d\left|a_{2}\right| d\left|b_{2}\right| \int \frac{d^{4} p}{(2 \pi)^{4}} \frac{1}{\left(k_{1}-p\right)^{2}} \frac{1}{p^{2}} \frac{1}{k_{3}^{2}} \\
& \exp \left(-i p \cdot\left(a_{2}-b_{2}\right)\right)\left(1-\exp \left(i k_{3} \cdot\left(C k_{2}+b_{2}\right)\right)\right) \\
= & V \frac{i \lambda^{3}}{m^{2}} \exp (i \Phi) \cos ^{2}\left(\theta_{1}\right) \cot \left(\theta_{3}\right) C k_{1} C k_{2} C \int d\left|a_{2}\right| d\left|b_{2}\right| \int \frac{d^{4} p}{(2 \pi)^{4}} \frac{1}{\left(k_{1}-p\right)^{2}} \frac{1}{p^{2}} \frac{1}{k_{3}^{2}} \\
& \left\{\exp \left(-i p \cdot\left(a_{2}-b_{2}\right)\right)\right. \\
& \left.-\exp \left(i\left(k_{1}-p\right) \cdot\left(a_{2}-b_{2}\right)-i k_{1} \cdot C k_{2}\right)\right\} \\
= & V \frac{\lambda^{3}}{m^{2}} \exp (i \Phi) \cos { }^{2}\left(\theta_{1}\right) \cot \left(\theta_{3}\right) C k_{1} C k_{2} C \int d\left|b_{2}\right| \int \frac{d^{4} p}{(2 \pi)^{4}} \frac{1}{\left(k_{1}-p\right)^{2}} \frac{1}{p^{2}} \frac{1}{k_{3}^{2}} \\
& \left\{\exp \left(i p \cdot b_{2}\right)\left(\exp \left(-i p \cdot C k_{1}\right)-1\right) \frac{C k_{1}}{p \cdot C k_{1}}\right. \\
& \left.+\exp \left(-i\left(k_{1}-p\right) \cdot b_{2}-i k_{1} \cdot C k_{2}\right)\left(\exp \left(i\left(k_{1}-p\right) \cdot C k_{1}\right)-1\right) \frac{C k_{1}}{\left(k_{1}-p\right) \cdot C k_{1}}\right\}
\end{aligned}
$$




$$
\begin{aligned}
= & V \frac{\lambda^{3}}{i m^{2}} \cos ^{2}\left(\theta_{1}\right) \cot \left(\theta_{3}\right) C k_{1} C k_{2} C \int \frac{d^{4} p}{(2 \pi)^{4}} \frac{1}{\left(k_{1}-p\right)^{2}} \frac{1}{p^{2}} \frac{1}{k_{3}^{2}} \\
& \left\{\exp \left(\frac{i}{2} k_{1} \cdot C k_{2}\right) \frac{C k_{1} C k_{2}}{p \cdot C k_{1} p \cdot C k_{2}}\left(1-\exp \left(-i p \cdot C k_{1}\right)\right)\left(1-\exp \left(-i p \cdot C k_{2}\right)\right)\right. \\
& -\exp \left(-\frac{i}{2} k_{1} \cdot C k_{2}\right) \frac{C k_{1} C k_{2}}{\left(k_{1}-p\right) \cdot C k_{1}\left(k_{1}-p\right) \cdot C k_{2}} \\
& \left.\times\left(1-\exp \left(i\left(k_{1}-p\right) \cdot C k_{1}\right)\right)\left(1-\exp \left(i\left(k_{1}-p\right) \cdot C k_{2}\right)\right)\right\} .
\end{aligned}
$$

In the first term of the above expression, we notice the following factor

$$
\int \frac{d^{4} p}{(2 \pi)^{4}} \frac{C k_{1} C k_{2}}{p^{2} p \cdot C k_{1} p \cdot C k_{2}} .
$$

Due to the additional propagator $1 /\left(k_{1}-p\right)^{2}$ in the full expression, the large momentum cut-off scale is $k_{1}$. The small momentum cut-off can also be seen to be $O(1 / C k)$. Therefore it could give rise to a large factor of $O\left(\lambda \log \left(C k^{2}\right)\right)$ in the large $k$ limit. It can be regarded as the correction to the first term of the tree amplitude in eq.(3.12). It can be associated with a corner of the first triangle. The second term is just analogous after the change of the variables from $\left(k_{1}-p\right)$ to $p$. It is the correction to the second term in eq.(3.12). It can also be associated with a corner of the second triangle. Since each loop gives rise to an additional logarithmic factor in the ladder diagrams, we need to sum them to all orders in the leading log approximation.

For this purpose we consider a generic ladder diagram with $n$ propagators around a corner of a triangle

$$
\begin{aligned}
& \int d^{4} x \int d^{4} x_{1} \exp \left(i k_{1} \cdot x_{1}\right) \\
& P \int_{C_{1}} d a_{n} \cdot A\left(x+x_{1}+a_{n}\right) \star \cdots \star \int_{C_{1}} d a_{2} \cdot A\left(x+x_{1}+a_{2}\right) \star \int_{C_{1}} d a_{1} \cdot A\left(x+x_{1}+a_{1}\right) \\
& \star \int_{C_{2}} d b_{1} \cdot A\left(x+b_{1}\right) \star \int_{C_{2}} d b_{2} \cdot A\left(x+b_{2}\right) \star \cdots \star \int_{C_{2}} d b_{n} \cdot A\left(x+b_{n}\right) \\
& \cdots \\
= & \frac{1}{m} \int d^{4} x \int d^{4} x_{1} \exp \left(i k_{1} \cdot x_{1}\right) \\
& \times \int_{C_{1}} d a_{1} \cdot \int_{C_{2}} d b_{1} \int_{C_{1}} d a_{2} \cdot \int_{C_{2}} d b_{2} \cdots \int_{C_{1}} d a_{n} \cdot \int_{C_{2}} d b_{m} \\
& \frac{\lambda}{4 \pi^{2}\left(x_{1}+a_{1}-b_{1}\right)^{2}} \frac{\lambda}{4 \pi^{2}\left(x_{1}+a_{2}-b_{2}\right)^{2}} \cdots \frac{\lambda}{4 \pi^{2}\left(x_{1}+a_{n}-b_{n}\right)^{2}} \\
& \cdots \\
= & V \frac{\lambda^{n}}{m} \int_{C_{1}} d a_{1} \cdot \int_{C_{2}} d b_{1} \int_{C_{1}} d a_{2} \cdot \int_{C_{2}} d b_{2} \cdots \int_{C_{1}} d a_{n} \cdot \int_{C_{2}} d b_{n} \\
& \int \frac{d^{4} p_{1}}{(2 \pi)^{4}} \frac{\exp \left(i p_{1} \cdot\left(a_{1}-b_{1}\right)\right)}{p_{1}^{2}} \int \frac{d^{4} p_{2}}{(2 \pi)^{4}} \frac{\exp \left(i p_{2} \cdot\left(a_{2}-b_{2}\right)\right)}{p_{2}^{2}} \cdots \int \frac{d^{4} p_{n}}{(2 \pi)^{4}} \frac{\exp \left(i p_{n} \cdot\left(a_{n}-b_{n}\right)\right)}{p_{n}^{2}}
\end{aligned}
$$




$$
\begin{aligned}
& (2 \pi)^{4} \delta\left(k_{1}-\sum_{i} p_{i}\right) \\
& \ldots .
\end{aligned}
$$

Here we may fix $a_{1}=b_{1}=0$ by the cyclic symmetry of the Wilson line. In the leading log approximation, we consider the contributions from the phase space where the momenta are strongly ordered.

$$
k_{1} \sim p_{1}>>p_{2}>>\cdots>>p_{n}
$$

In real space, it corresponds to the following region

$$
1 / k_{1} \sim x_{1}<<a_{2}-b_{2}<<\cdots<<a_{n}-b_{n}
$$

Let us assume that we get logarithmic factors for each leg of the ladder as we will find shortly. Let us assume that the scale of the legs of the ladder is uniformly distributed in the logarithmic scale over $\log (L)$. We can then estimate the amplitude with $n$ legs as $(\log (L) / n)^{n} \sim \log ^{n}(L) / n$ !. Therefore these characteristic log factors in the leading $\log$ approximation imply that the scale of the legs is uniformly distributed in the logarithmic scale. The uniform distribution in the logarithmic scale corresponds to strongly ordered distributions in phase space eq.(3.17) or in real space eq.(3.18). With such an approximation, the above expression can be evaluated as

$$
\begin{aligned}
& \lambda^{n} \cos \left(\theta_{1}\right)^{n} \frac{1}{k_{1}^{2}} C k_{1} C k_{2} \\
& \int_{|a|_{1}}^{C k_{1}} d|a|_{2} \int d|b|_{2} \frac{1}{4 \pi^{2}\left(a_{2}-b_{2}\right)^{2}} \cdots \int_{|a|_{n-1}}^{C k_{1}} d|a|_{n} \int d|b|_{n} \frac{1}{4 \pi^{2}\left(a_{n}-b_{n}\right)^{2}} \\
& \ldots .
\end{aligned}
$$

Our strategy is to perform integrations over $b$ next. Although we have to deal with nested integrations, the problem simplifies due to the strong ordering. Let us consider a particular leg of the ladder. Then the inner legs are much shorter and the outer legs are much longer than it. So effectively we can shrink all the inner legs to the point and move the outer legs to the infinity. Therefore we are left with the following single integral

$$
\begin{aligned}
\int d|b| \frac{1}{(a-b)^{2}} & =\int_{-|a| \cos \left(\theta_{1}\right)}^{\infty} d b \frac{1}{|a|^{2} \sin \left(\theta_{1}\right)^{2}+|b|^{2}} \\
& =\frac{1}{|a| \sin \left(\theta_{1}\right)} \int_{-\cot \left(\theta_{1}\right)}^{\infty} d b \frac{1}{1+b^{2}} \\
& =\frac{1}{|a| \sin \left(\theta_{1}\right)}\left(\pi-\theta_{1}\right) .
\end{aligned}
$$


After integrating over $|b|$ variables in this way, we obtain

$$
\begin{aligned}
& \left(\frac{\lambda}{4 \pi^{2}}\right)^{n} \cot \left(\theta_{1}\right)^{n-1} \cos \left(\theta_{1}\right)\left(\pi-\theta_{1}\right)^{n-1} \frac{1}{k_{1}^{2}} C k_{1} C k_{2} \\
& \int_{1 / k_{1}}^{C k_{1}} d\left|a_{2}\right| \frac{1}{\left|a_{2}\right|} \cdots \int_{\left|a_{n-1}\right|}^{C k_{1}} d\left|a_{n}\right| \frac{1}{\left|a_{n}\right|} \\
& \ldots
\end{aligned}
$$

Since $|x|<\left|a_{2}\right|<\cdots<\left|a_{n}\right|<k_{1}$, the integration ranges are easy to understand. With these justifications, the above integral is found to be

$$
\begin{aligned}
& \left(\frac{\lambda}{4 \pi^{2}}\right) \cos \left(\theta_{1}\right) \frac{1}{k_{1}^{2}} C k_{1} C k_{2} \\
& \left(\frac{\lambda}{4 \pi^{2}} \cot \left(\theta_{1}\right)\left(\pi-\theta_{1}\right) \log \left(C k_{1}^{2}\right)\right)^{n-1} \frac{1}{(n-1) !} \\
& \ldots
\end{aligned}
$$

After summing over $n$, we obtain the power enhancement factor in association with a corner

$$
\exp \left(\frac{\lambda \cot \left(\theta_{1}\right)\left(\pi-\theta_{1}\right)}{4 \pi^{2}} \log \left(C k_{1}^{2}\right)\right)
$$

It is clear that such a power law can be associated with each corner of the Wilson loops formed by the Wilson lines. I Such corner divergences are well known to occur in the Wilson loop expectation values. It is also studied in ordinary gauge theory through $A d S / C F T$ correspondence[17]. The appearance of such a power law enhancement in the Wilson line correlators in the high energy limit which is characteristic to the Wilson loops is consistent with our assertion that they are indeed equivalent.

\section{Conclusions and Discussions}

In this paper we have shown that high energy behavior of the correlators of the Wilson lines is identical to the expectation value of large Wilson loops. In the case of three point functions, the dominant region of the phase space in eq.(3.17) corresponds to the configuration such that $x_{1}=x_{3}=1 / k$ since the propagators which are localized at the two corners of the triangle are of the length of $1 / k$. The Wilson lines effectively form a closed loop in such a region of the phase space. Therefore the length of the segments which do not involve

\footnotetext{
${ }^{2}$ The possibility of such a power law enhancement between nearly parallel Wilson lines is independently noted by 18.
} 
gauge fields are vanishingly small. So we expect that their effect is equivalent to insertion of local operators in the closed Wilson loops. If so, they do not influence the universal large momentum behavior of the correlators. These arguments also apply to multi-point functions of Wilson lines. We therefore find that the high energy limit of the Wilson line correlators can be described by large closed Wilson loops in the weak coupling region.

In the high energy limit in the weak coupling regime, the multi-point correlation functions of the normalized Wilson lines behave as

$$
\begin{aligned}
& <W\left(k_{1}\right) W\left(k_{2}\right) \cdots W\left(k_{n}\right)> \\
\sim & \exp \left(-\sum_{i=1}^{i=n} \sqrt{\frac{\lambda}{4 \pi}\left|C k_{i}\right|\left|k_{i}\right|}+\sum_{i=1}^{i=n} \frac{\lambda \cot \left(\theta_{i}\right)\left(\pi-\theta_{i}\right)}{4 \pi^{2}} \log (|C k||k|)\right)
\end{aligned}
$$

where $\theta_{i}$ is the $i$-th angle of the relevant closed Wilson loop and we assume that $k_{i}$ are all of the same order $k$. In this expression, the exponential suppression of the normalized multi-point function is caused by the exponential enhancement of the two point function.

It is conceivable that the equivalence of the Wilson lines and Wilson loops at high energy continues to hold at strong coupling. We may be able to prove it by making the loop equation argument precise. Here we would like to refer to our work where the Wilson loop expectation value in NCYM has been investigated by Nambu-Goto action [13. There we consider superstring theory in a particular background. The string frame metric possess the maximum at the scale $R \sim(\lambda)^{1 / 4}$ in the fifth radial coordinate $r$. We have proposed to put the Wilson loops at $r=R$. In such a construction, we obtain analogous expression with $A d S / C F T$ correspondence since the relevant Wilson loops are large. The only novelty is to identify the short-distance cut-off with $R$. With this prescription we predict the strong coupling behavior of the Wilson lines as follows:

$$
\begin{aligned}
& <W\left(k_{1}\right) W\left(k_{2}\right) \cdots W\left(k_{n}\right)> \\
\sim & \exp \left(-R \sum_{i=1}^{i=n} \sqrt{\left|C k_{i}\right|\left|k_{i}\right|}+R^{2} \sum_{i=1}^{i=n} \frac{\cot \left(\theta_{i}\right)\left(\pi-\theta_{i}\right)}{\pi} \log \left(|C k||k| / R^{2}\right)\right) .
\end{aligned}
$$

It is because $\sum_{i=1}^{i=n} \sqrt{\left|C k_{i}\right|\left|k_{i}\right|}$ is proportional to the perimeter length of the Wilson loop in fully noncommutative gauge theory.

We recall here that the average distance of the two point function is $O\left(R^{2}\right)$ with respect to the noncommutativity scale in the weak coupling limit as it is explained in section 3. The standard prescription $R^{2} \rightarrow R$ may imply that the minimal length scale which can be probed by the high energy limit of the two point function is indeed $R$ in the strong coupling 
limit. It is hence likely that our prescription to put the Wilson loops at $r=R$ is relevant in such a limit. The $\log (|C k||k|)$ behavior in the weak coupling expression in eq.(4.1) is already remarkable in that the short distance cut-off $1 / k$ and the long distance cut-off $C k$ are related through the noncommutativity scale $C$. It is reminiscent of $T$ duality in string theory. However eq.(4.2) predicts the appearance of $R^{2}$ factor in the logarithm in the strong coupling limit.

\section{Acknowledgments}

This work is supported in part by the Grant-in-Aid for Scientific Research from the Ministry of Education, Science and Culture of Japan. 


\section{References}

[1] T. Banks, W. Fischler, S.H. Shenker and L. Susskind, M-theory as a matrix model: a conjecture, Phys. Rev. D55 5112 (1997); hep-th/9610043.

[2] N. Ishibashi, H. Kawai, Y. Kitazawa and A. Tsuchiya, A Large-N Reduced Model as Superstring, Nucl. Phys. B498 (1997) 467; hep-th/9612115.

[3] A. Connes, M. Douglas and A. Schwarz, JHEP 9802: 003.1998; hep-th/9711162.

[4] H. Aoki, N. Ishibashi, S. Iso, H. Kawai, Y. Kitazawa and T. Tada, Noncommutative Yang-Mills in IIB matrix model, Nucl. Phys. 565 (2000) 176; hep-th/9908141.

[5] N. Ishibashi, S. Iso, H. Kawai and Y. Kitazawa, Wilson loops in non-commutative YangMills, Nucl. Phys. B573 (2000) 573; hep-th/9910004.

[6] N. Ishibashi, S. Iso, H. Kawai and Y. Kitazawa, String scale in non-commutative YangMills, Nucl. Phys. B583 (2000) 159; hep-th/0004038.

[7] J. Ambjorn, Y. Makeenko, J. Nishimura and R. Szabo, Finite $N$ matrix models of noncommutative gauge theory, JHEP 9911 (1999) 029, hep-th/9911041; Non-perturbative dynamics of non-commutative gauge theory, Phys. Lett. B480 399, hep-th/0002158; Lattice gauge fields and discrete non-commutative Yang-Mills theory, JHEP 0007 (2000) 013, hep-th/0003208.

[8] M. Fukuma, H. Kawai, Y. Kitazawa and A. Tsuchiya, String field theory from IIB matrix model, Nucl. Phys. B510 (1998)158, hep-th/9705128.

[9] N. Seiberg and E. Witten, String theory and noncommutative geometry, JHEP 9909 (1999) 032; hep-th/9908142.

[10] R. Gopakumar, S. Minwalla and A. Strominger, Noncommutative solitons; JHEP 0005 (2000) 020; hep-th/0003160.

[11] D. J. Gross, A. Hashimoto and N. Itzhaki, Observables of Non-Commutative Gauge Theories; hep-th/0008075.

[12] A. Dhar and S. R. Wadia, A Note on Gauge Invariant Operators in Noncommutative Gauge Theories and The Matrix Model; hep-th/0008144. 
[13] A. Dhar and Y. Kitazawa, Wilson loops in strongly coupled noncommutative gauge theories; hep-th/0010256.

[14] H. Liu, *-Trek II; hep-th/0011125.

[15] S. R. Das and S. P. Trivedi, Supergravity coupling to Noncommutative Branes, Open Wilosn Lines and Generalized Star Products; hep-th/0011131.

[16] M. Abou-Zeid and H. Dorn, Dynamics of Wilson Observables in Non-commutative Gauge Theory; hep-th/0009231.

[17] N. Drukker, D. J. Gross and H. Ooguri, Wilson Loops and Minimum Surfaces; hepth/9904191.

[18] M. Rozali and M. V. Raamsdonk, Gauge Invariant Correlators in Non-Commutative Gauge Theory; hep-th/0012065.

[19] Soo-Jong Rey and R. von Unge, S-duality, non-critical open string and non-commutative gauge theory; hep-th/0007089, S. J. Rey and S. R. Das, Open Wilson Lines in Noncommutative Gauge Theory and Tomography of Holographic Dual Supergravity; hepth/0008042.

[20] T. Eguchi and H. Kawai, Phys. Rev. Lett. 48 (1982) 1063.

G. Parisi, Phys. Lett. 112B (1982) 463.

D. Gross and Y. Kitazawa, Nucl. Phys. B206 (1982) 440.

G. Bhanot, U. Heller and H. Neuberger, Phys. Lett. 113B (1982) 47.

S. Das and S. Wadia, Phys. Lett. 117B (1982) 228.

[21] A. Gonzalez-Arroyo and M. Okawa, Phys. Lett. B120 (1983) 174, Phys. Rev D27, (1983) 2397.

A. Gonzalez-Arroyo and C. P. Korthals Altes, Phys. Lett. 131B,396 (1983).

[22] T. Eguchi and R. Nakayama, Phys.Lett. B126 (1983) 89

T. Filk, Phys. Lett. B376 (1996) 53 .

[23] M. Li, Nucl.Phys. B499 (1997) 149;hep-th/961222. 